\title{
The dissemination of news in Social Communication Networks Chen zhengyifan
}

\author{
School of North China Electric Power University, Baoding 071000, China \\ 519604267@qq.com
}

Keywords: Small World Network Model, The dissemination of news Social Communication Networks

\begin{abstract}
Public interest and opinion can be changed by news. So, it is necessary to study the The dissemination of news. In this paper, an improved Small World network model is introduced to simulate the dissemination of information so that the characteristics of the dissemination of information can be figured out. Low average distance and high clustering coefficient are two main attractive properties of small-word network. Based on the simulation ,we draw a conclusion that Information Diffusion is due to the inherent value of the information itself, as well as the news finding its way to influential or central network nodes that accelerate its spread through social media.
\end{abstract}

\section{Introduction}

News is packaged information about current events happening somewhere else. News moves through many different media, based on word of mouth, printing, postal systems, broadcasting, and electronic communication. News is spread quickly in today's tech-connected communication network and we want to know more about the characteristic of the dissemination of information.In order to study the we build a small-world network model to, regarding the participants as nodes, and taking the communication among participants as sides.

\section{Small-World Network Model ${ }^{[1]}$}

A small-world network is a type of mathematical graph in which most nodes are not neighbors of one another, but most nodes can be reached from every other node by a small number of hops or steps. Specifically, a small-world network is defined to be a network where the typical distance L between two randomly chosen nodes (the number of steps required) grows proportionally to the logarithm of the number of nodes $\mathrm{N}$ in the network, that is $L \propto \log N$. In the context of a social network, this results in the small world phenomenon of strangers being linked by a short chain of acquaintances. Many empirical graphs show the small-world effect, e.g., social networks, the underlying architecture of the Internet, wikis such as Wikipedia, and gene networks.

\section{Some Basic Concepts of our Small-world networks ${ }^{[2]}[3]$}

Edges: An edge is the line between two nodes interconnected. Focused on the present network that regard participants as points, the two nodes that are connected together in by an edge have the opportunity to pass information to each other.

Nodes: The nodes in the network model Node in the network represents the participants in the social communication network. It should be noted that those nodes not only refer to the very person, but also the media organizations and information exchange transfer station in the communication networks. The total number of nodes is represented by $N$

Neighbor: the most close nodes to the target node.

The degree or connectivity of a node: The degree or connectivity of a node is the number of neighbors it has. Using the adjacency matrix, we can formally define the degree of a node as: 


$$
k_{i}=\sum_{j \in N} a_{i j}
$$

If the graph is directed, then $k_{i}$ will have two components: the ingoing links $k_{i}^{i n}=\sum_{j} a_{i j}$ and the outgoing links $k_{i}^{\text {out }}=\sum_{j} a_{i j}$ the total degree will be $k_{i}=k_{i}^{\text {out }}+k_{i}{ }^{\text {in }}$.

Degree distribution.:We can define the degree distribution of the graph, $\mathrm{P}(\mathrm{k})$, as the fraction of nodes in the network that have connectivity, or equivalently, the probability that a node randomly chosen from the network has k neighbors.

The value of news:The information is divided into different areas, generally about political, econo mic, cultural and technological. Set the attentions of every areas to $\mu_{\mathrm{i}}$

$$
\mu_{\mathrm{i}}=\frac{N}{M}
$$

$N$-A month's pageviews of a web page about the every area .

$M$-The total amount of pageviews of all the web pages

Except the heat in the field of Journalis. The value of the news is also related to its credibility $\beta$ reliability depends on the specific circumstance. So the value of a news can be described as $\eta_{i}$ :

$$
\eta_{i}=\beta \times \mu_{i}
$$

\section{The principles of the dissemination of information}

The node in our small-world network has a parameter $\omega_{i}$ which is used to characterize the minimum requirement for the dissemination of information. If a node got a message from one of its neighbors, it will make a decision whether to disseminate this information to its neighbors based on the quantitative relationship between $\eta_{i}$ and $\omega_{i}$.

If $\eta_{i}>\omega_{i}$, information is passed to other neighboring nodes.

If $\eta_{i}<\omega_{i}$, information, information will not continue to spread in this cycle. 


\section{Simulation of the flow of information}

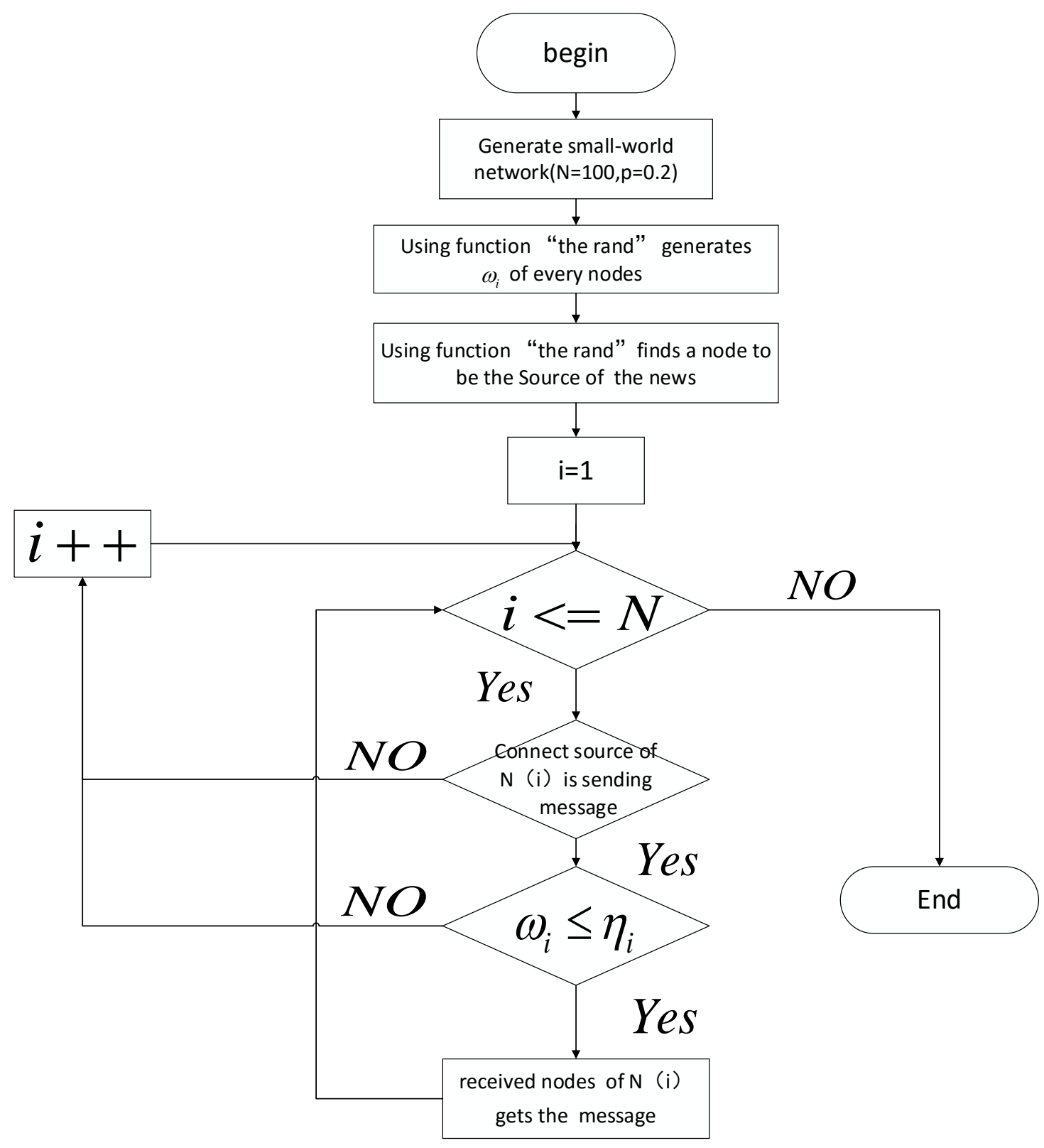

\section{Result \&Analysis}

We put the different kinds of the information into our small-world network and simulate the ways of information dissemination. There are different kind of information whose value is $a, b$ and $c$. Regarding every code as the source of information, the average time of information spreading in the network. The result of simulation is shown as Figure 2.The information whose value is smaller is spread slower, while the information whose value is greater is spread fastest. Putting the same information into three different nodes, the simulation is shown as Figure 2. The algorithm of information spread is shown as Figure 3

We suspect that the speed of information dissemination is also connected with the attributes of the source information. 


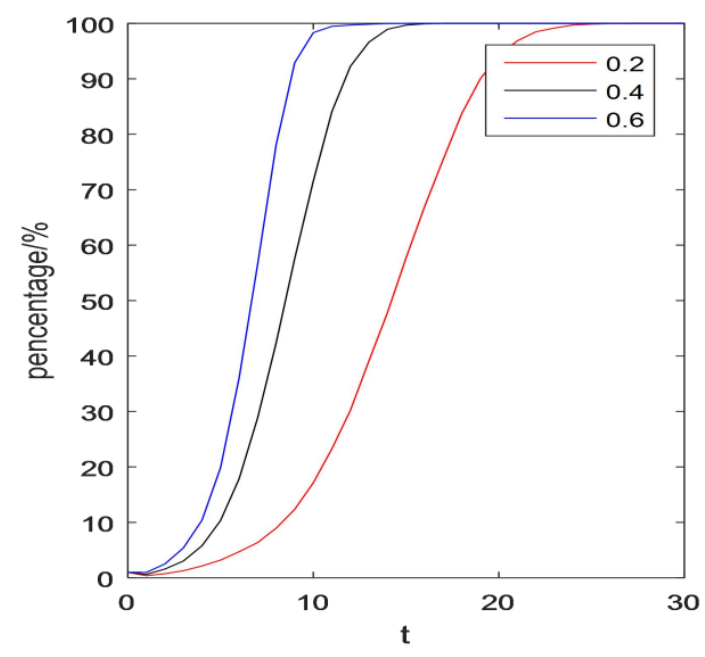

Figure 2

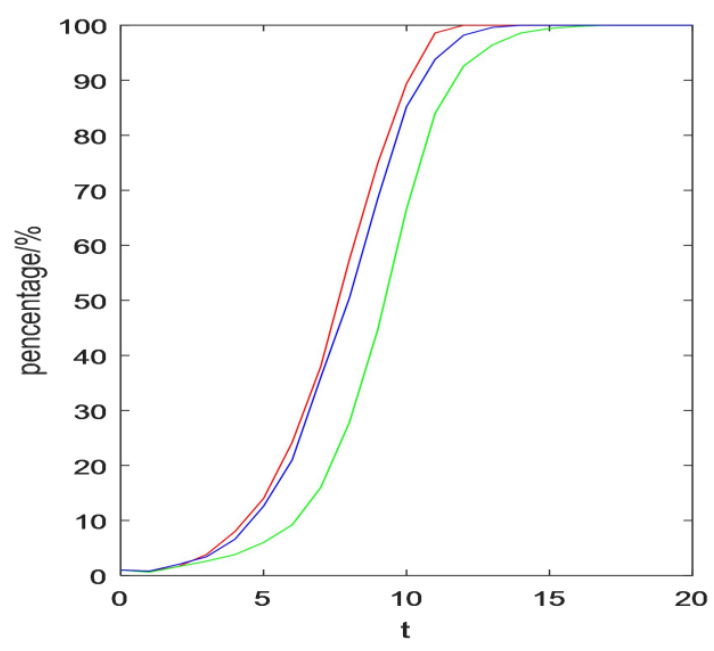

Figure 3

Different information put into small-word networkThe same information put into different source

\section{Summary}

Conclusion has been drawn that the more important the information is, the faster it will spreading in the small-word social network, which is consistent with our common sense. The more valuable information disseminates faster in the network.

\section{References}

[1] https://en.wikipedia.org/wiki/Small-world_network

[2] Ted G. Lewis. NETWORK SCIENCE: Theory and Applications [M]. New York: A JOHN WILEY \& SONS, INC, 2009

[3] http://www.ee.cityu.edu.hk/ gchen/pdf/CW-CASM03-overview.pdf 